\title{
On Teaching Model-based Fault Diagnosis in Engineering Curricula
}

Control and systems theory is a common subject in many engineering curricula. Students learn from the very basics of system modeling to the most recent advances in control algorithms. However, although fault diagnosis of dynamic systems is already a mature and an important field, it is not usually included in most engineering curricula.

The level of industrial automatization is increasing as well as the complexity of supervisory tasks. In most plants, these tasks are still performed by human operators through supervisory control and data acquisition (SCADA) systems. Operators should react by taking appropriate corrective actions when a variable exceeds the safety threshold. Automatic supervision systems that include fault diagnosis capabilities to help operators to take corrective actions could be extremely useful in complex plants. However, despite significant academic theoretical advancements, industrial implementation lags behind. In part this is due to the fact that most engineering students are not aware of the existence of fault-diagnosis methodologies. To let students know about these methodologies, fault diagnosis should be introduced in engineering curricula at undergraduate or Master's level. This article is a step towards that direction. 


\section{Context}

Among the fault diagnosis methodologies, model-based fault diagnosis is the best developed from a conceptual point of view [1]. Moreover, this fault-diagnosis approach is closely linked to systems theory and control knowledge that engineering students already have. For this reason, the model-based fault diagnosis is the core of the course Diagnosis and Fault-Tolerant Control in the Automatic Control and Robotics Master's degree program at Universitat Politècnica de Catalunya (UPC) described in this article.

Model-based fault diagnosis of dynamic systems is based on the use of models to check the consistency between observed and modeled behaviors (Figure 1). Model-based methods rely on analytical redundancy achieved by the use of a mathematical model that combines measurements from other correlated sensors (spatial redundancy) or from the same sensor in past time instants (temporal redundancy). Alternatively, hardware redundancy is based on the use of redundant (extra) sensors. The consistency check in model-based methods is based on computing the difference between the predicted values (from the model) and measured ones (from sensors). This difference, known as the residual, is compared with a threshold value (zero in the ideal case); when this residual is bigger than the threshold, it is determined that there is a fault in the system, for details see "Faults". Otherwise, the system is considered to be functioning properly. Fault detection is followed by fault isolation, which distinguishes a particular fault from the others, for details see "Faults Diagnosis". Although a single residual is sufficient to detect faults, a set (or a vector) of residuals is required for fault isolation [2]. If a fault is distinguishable from other faults using a residual set, then it can be said that this fault is isolable.

The first model-based fault-diagnosis methods were presented in [3], for details see "Modelbased Fault Detection and Isolation". Since then, a huge amount of research has been carried 
out resulting in many different methods. These methods can be classified as: parity methods [2], observer methods [4], and parameter-estimation methods [5], [6]. Mathematical relations among these methods have been established by several authors and are now well understood (see for example [2]) where the conditions under which they provide equivalent results are given.

\section{Learning Outcomes and Course Sylabus}

According to the Master structure, the course is elective in the last semester with 4,5 European Credit Transfer and Accumulation System (ECTS). ECTS is the standard unit in the European university system. Each ECTS corresponds to 10 hours of guided activities (lectures or laboratory session in our case) plus 20 hours of student work, individually or in group.

When defining the course contents, professors discussed about which were the students prerequisites to successfully address the course. By analyzing model-based fault diagnosis fundamentals, the professor team agreed that they could be grouped in two main blocks. A first block corresponding to linear systems theory including transfer-function and state-space formalisms, stability and observability analyses and methods such as Luenberger observer design and linearization of nonlinear systems around an equilibrium point. The second block includes system modeling, identification and simulation using numerical methods. All these concepts and methods are provided in previous courses and are assumed known at the beginning of the course. Another major issue was the definition of the set of learning outcomes (SLO), that esblablishes what the professors expect that the students should know, understand or be able to do if they successfully complete the course. After analyzing the fault diagnosis literature, the professors 
agreed that on successful completion of the course, students should be able to:

SLO1 recognize the principles of model-based fault-diagnosis (see Figure 1) and its components in a real FDI system.

SLO2 derive analytical redundancy relations using the structural analysis to generate a set of structured residuals from the model equations as well as its use for sensor placement to the design of a fault-diagnosis system (see "Structural Analysis and Sensor Placement") through the use of SaTool [1] in a real case study (see Figure 6).

SLO3 analyze the fault detectability and isolability properties of the residuals using the fault signature matrix (FSM) (see Table III) in a particular case.

SLO4 apply the most-used techniques (parity equations and observers) to a particular system.

SLO5 design a set of structured residuals that satisfy some fault isolation specifications regarding a set of pre-established faults (see Table III) in a real case study.

SLO6 distinguish the differences between the simulated and real behavior in a laboratory experience which resembles a real industrial application.

SLO7 apply system-identification techniques to perform the fault estimation and the use of the obtained estimation to implement fault-tolerance techniques based on virtual sensors/actuators in a real case study.

SLO8 work as part of a team in analyzing and developing a fault diagnosis project.

Having all this in mind the syllabus of the course was defined as follows:

1) Introduction. In this section of the course, the need of fault diagnosis in industrial systems is motivated. An overview of existing methodologies is provided. Signal/data and knowledge based methods are shortly revised.

2) Structural Analysis and Sensor Placement. Structural analysis to determine a set of 
analytic redundancy relations consistent with the system model structure and the available measurements is introduced. The problem of sensor placement for fault diagnosis is presented and ways of addressing it are described.

3) Fault Detection. From the set of analytic redundancy relations derived from the structural analysis, several ways of implementing the residuals are discussed. Parity equations and observer-based methods, and their relationship are presented in detail. The need of robustness against uncertainty, noise and disturbances is introduced. Statistical and setmembership methodologies are discussed as ways to handle these problems.

4) Fault Isolation. Fault isolation from the analytic redundancy relations is presented. Two approaches are discussed: the one coming from the automatic control (FDI) community and the one coming from the model-based fault diagnosis (DX) community in the artificial intelligence area. Recent results of the BRIDGE approach [7] that link these two approaches are also introduced.

5) Fault Estimation. Fault estimation is introduced as the last step of the fault-diagnosis cycle. Once the fault has been detected and isolated, the fault can be estimated. Several approaches based on parameter estimation and different types of observers are presented.

6) Fault-tolerant Control. An introduction to fault-tolerant control as a direct application of fault diagnosis is presented. The most relevant strategies of fault-tolerant control are described including the reconfiguration/accommodation of the faulty control system and the use of virtual sensors and actuators [1].

7) Case studies. Several real case studies based on the experience of the Advanced Control Systems (SAC) group of the UPC in different projects are presented.

According to the syllabus and the SLOs, the 45 hours of guided activities were decomposed 
on 30 hours of theoretical lectures plus 12 hours of experimental laboratories. In order to fit appropriately in the Master time table, the labs sessions are organized in blocks of 3 hours.

\section{Teaching materials}

Although there is a huge amount of research articles and books on these topics, the amount of material that can be used for teaching purposes is not very large. Although survey articles $[7]-[15]$ might be used as a starting point, they are not comprehensive enough for teaching purposes.

Books usually offer a more consistent and comprehensive formulation being a better option for teaching purposes. For many years, there was only one book [16]. Currently, other books exist in this area, [2] mainly addresses the problem of fault diagnosis using the parity equation approach, [17] focuses on the observer approach with an explicit emphasis to the problem of robustness, [1] deals with the problem of diagnosis and fault-tolerant control starting from structural analysis using either classical model based or qualitative approaches, [6] provides a tutorial introduction to fault diagnosis and fault-tolerant systems using several methodologies and real world applications, [18] is a second book from the same author that shows how the methods introduced in [6] can be applied for a selection of twenty real technical components and processes, and finally [19] provides an introduction to basic model-based fault-diagnosis schemes, advanced analysis and design algorithm; the book also provides a comparison among different methods.

Maybe [6] is the one that offers the widest perspective since it presents a wide range of faultdiagnosis methods covering model-based, signal-based, and knowledge-based methods. It is a book that can be used to teach fault diagnosis even to undergraduate students since the level of 
mathematics and the required system theory is low. Morevover, it includes exercises at the end of each chapter. On the other hand, the other books are more convenient for graduate students who are working towards their Master's or $\mathrm{PhD}$ thesis in fault diagnosis.

Another important element to support teaching are computer tools. As in other control fields, many toolboxes have emerged recently. Most of them can be easily adapted for teaching purposes. The most relevant ones are:

- SaTool [20] is a tool to analyze the system structure at a high level. SaTool offers a Matlabbased GUI that allows analyzing detectability and isolability, and deriving the analytic redundancy relations for the normal and faulty cases selected by the user.

- Fault Detection Toolbox [21] provides a comprehensive set of high-level Matlab functions to support the design of residual generation filters using reliable numerical algorithms. The basic computational layer is formed by the Descriptor Systems toolbox [22], which contains all necessary tools to solve the underlying numerical problems.

- FDI-Toolbox [23] allows the design of fault-diagnosis systems using observer-based and parity-space FDI. The development of the FDI-Toolbox is based on Matlab and the Control Systems and Robust Control Toolboxes.

\section{Lab sessions}

\section{Framework}

When preparing the laboratory sessions professors discussed possible teaching methodologies to achieve the proposed learning goals. So far, laboratory sessions in most Master's courses had been organized in quite a standard way. There was a student guide for the experiments and a description of the results that the student should obtain. Moreover, the labs were done in a 
straightforward manner working with simulated or very simplified experimental environments. After some discussions, professors agreed that students "should learn by doing" and that "realworld problems" capture students' interest, while at the same time the students acquire and apply new knowledge in a "problem-solving context". This is in fact the key element the project-based learning (PBL) methodology is focused on, "Project-Based Learning" contains more information about this. For this reason, the lab activities associated to the fault diagnosis course use a PBL methodology [24] $-[26]$. A concrete problem is formulated and students must provide a solution based on the theoretical contents seen in the theoretical lectures. They must solve the problem in a situation which is quite similar to that they will find in the real work. As the course is taken in parallel with other courses and experimental sessions are limited to 12 hours, it is important that the students provide a solution in time so they can take profit from the prescheduled laboratory sessions. Due to this the project is split in several consecutive goals the students must achieve. Each goal is expected to be addressed in one of the experimental sessions.

To develop the project, the students are grouped in groups of three or four students. Additionally the teacher helps the student team by discussing with them and helping to identify the correct solutions. This procedure allow students to complete the project within the predefined schedule. However the concrete solution is completely proposed and developed by the students.

Among the different techniques presented in the theoretical part of the course, professors have decided to select a set of activities that cover the whole design cycle of a FDI system. Firstly, a model for the system must be obtained, form this model a structural analysis must be performed. This analysis will allow to obtain the analytic redundancy relations to generate residuals able to detect and isolate the faults of interest. After, using observer-based approaches, the residuals and the fault isolation logic must be implemented; in this context it is necessary to understand 
that the observer gain can be used as a tuning parameter that changes the detection performance. Then, fault estimation techniques and virtual sensors/actuators fault-tolerant control techniques can be developed. Finally, the fault-diagnosis system must be tested in the real setup to assess the performance.

\section{Experimental setup}

A three-tank system was selected as the case study to apply the PBL methodology in the Master's course. Tank systems, both in three or four-tanks versions, have been widely used for teaching and research purposes [27] [28] [29] [30] [31] due to their interesting dynamic behavior and relatively simple construction.

Figure 5 shows the experimental setup in which real experiments are performed. This experimental setup has been built at the Automatic Control Department (ESAII) of the UPC to support experimental works in different subjects. It is constructed of polymethylmethacrylate. To measure the complete state, three ultrasonic sensors (Honeywell, 945/LAY-AD-1CO) at the top of each tank measure the distance from the top of the tank to the liquid level. Two peristaltic pumps (Flojet, 2100-740) are used as actuators since they have a linear behavior in a wide operation range. These pumps can move liquid from the bottom tank to any of the upper ones. The interconnection between the different tanks is made by means of flexible pipes and connectors, so the system can be easily reconfigured. Some of the pipes include a valve (Legris, 6401), which allows the pipe configuration to be modified. The tanks are filled with colored water to make experimental measurements easier and also include a ruler. The interconnection of this system with a computer running Matlab and Simulink is established via a device that provides the electronic power, adapts, and cleans the signal from the sensors. A setup simulator in Simulink is also available 
based on an accurate mathematical model of the real system. This simulation model is provided to the students so they can work at home, in the meantime between two laboratory sessions. As we only have one real setup, the simulation model allows to verify and properly tune the algorithms before real testing. All project steps are first validated in the simulation model; once each goal has been successfully achieved in the simulator, then algorithms are executed and validated in the real setup without needing to recode them. Students are evaluated from the results obtained in the real setup.

Changes in the valve section and sensor position are generated manually, so it is not possible to introduce very precise changes in the system, the simulator can be used instead if more precision is needed. This provides characteristics similar to the ones an engineer would find in a real environment. The faults to be detected and isolated are defined by the instructor during the project definition. Different groups might have different specifications.

The labs of the fault diagnosis course are organized in five sessions of three hours that are distributed along the course taking into account that required concepts have been previously introduced in the lectures. The last session is devoted to the students presentations of the results achieved in the project development. The remaining sessions are described in the following subsection with indication of the SLOs addressed.

\section{Experimental sessions}

\section{Structural Analysis and Sensor Placement}

The three-tank model is composed of the components presented in Table II. In this table, the equation that describes the dynamics of each component is also shown. From this set of equations, students perform the structural analysis of the system using SaTool [20]. As a result of this 
analysis, the structure of the system presented in Figure 6 is obtained. Using the perfect matching algorithm available in SaTool (see [20] for more details), the set of analyical redundancy relations from which a set of structured residuals is obtained as well as the FSM regarding the set of considered faults (see Table II). Finally, students perform the analysis of fault detectability and isolability of the obtained set of structured residuals and discuss about possible new sensors that could be added to improve these properties. This session contributes to the achievement of SLOs $1,2,3$ and 8.

Fault Detection using Observer and Parity Equations

In this session, students obtain a set of residuals derived from the analytic redundancy coming from the first lab session. Residuals are implemented using the parity equation and observer methodologies and are evaluated in different fault scenarios (leaks, sensor, and actuator faults) to check the sensitivity to different faults when varying the observer gain and changing the residual implementation from the ARMA-parity equation form to the MA-parity equation form (deadbeat observer) [32]. Figure 7 shows fault detection results obtained with the considered set of residuals when an offset fault appears in the third tank level sensor. This figure shows also the effect of varying the observer gain in the fault detection performance. SLOs 4,6 and 8 are related with this session.

\section{Fault Isolation using Structured Residuals}

Analyzing the set of obtained (primary) residuals in the first lab session and the set of considered faults, the FSM presented in Table $\Pi$ in obtained. In this session, this matrix is validated in simulation. The students should notice using the structural analysis approach that not all the residuals are isolable. Transforming this set of residuals by following the approach described in 
[2], a new set of residuals allows the desired set of faults to be isolated (see Table [III). This analysis is carried out using the FDI-Toolbox [23]. Finally, the students implement the fault isolation algorithm based on matching the observed residuals and the FSM presented in Table [II] which will be tested by introducing some artificial faults into the system. As an illustrative sample of the results that the students should obtain, Figure 7 presents the case when a fault appears in the third tank level sensor, it can be seen that only the third residual is activated. Thus, according to Table III, this fault can be isolated. SLOs 5, 6 and 8 are addressed in this laboratory session.

\section{Fault Estimation and Fault-tolerant Control}

The fault-diagnosis system implemented in previous sessions is enhanced by implementing an algorithm that allows the fault to be estimated. Using the information about the type of fault and the fault estimate, a virtual sensor scheme based on an observer is implemented that compensates for faults in sensor measurements. In the lab session, SLOs 6,7 and 8 are carried out.

\section{Assessment}

The assessment of the activities is organized around the three-tank PBL. The aim is to assess the learning process instead and the achievement of the different SLO rather than making a final knowledge evaluation. At the end of every lab session, students show to the professor how their developments work and deliver a report which justify how they obtained the algorithms and results. Then, professor proceeds to verify the report contents and to do an oral exam to the students to see if they have understood the results obtained, ensuring in this way, the continuous assessment. Together the lab session evaluations counted for $70 \%$ of the final mark. In the last 
lab session, each lab group has to present in front of the other students and the professors a summary of the project as well as a demonstration of how the fault diagnosis system works. After this presentation, professors do an oral exam to assess that students have learned the concepts that are part of the SLO of the course. This final evaluation of the overall project counted for $30 \%$ of the final mark.

Figure 2 presents the assessment results when PBL was not applied. On the other hand, Figure 3 shows the assessment results after the application of PBL methodology. As it can be seen, introducing the PBL methodology has improved the course results. Forcing the students to address all the steps in a fault-diagnosis project allows the students to clearly understand what is the role of each element. This clearly influences positively the evaluation results.

\section{Survey}

A survey was conducted to obtain feedback from the students at the end of the course. Each year, the course is taken by approximately between 8 and 10 students. Figure 4 contains a summary of students' opinions obtained through an anonymous survey. Two types of questions are made to the students, the first type is related to they feeling about the SLO achievement (questions $1,2,3$ and 4) while the second type is related to the tools used during the course (questions 5,6 and 7). Looking at the answers, it can be seen that according to the students perception they have acquired most of SLOs which is consistent with the assessment results. This feeling is a good way the improve the course learning environment and improve students' enthusiasm. Additionally, survey results show that students are satisfied with the exercises; they consider the PBL activities a good way to understand and identify all the components and algorithms involved in a fault-diagnosis system. Also, the proposed teaching material has been rated as good by most 


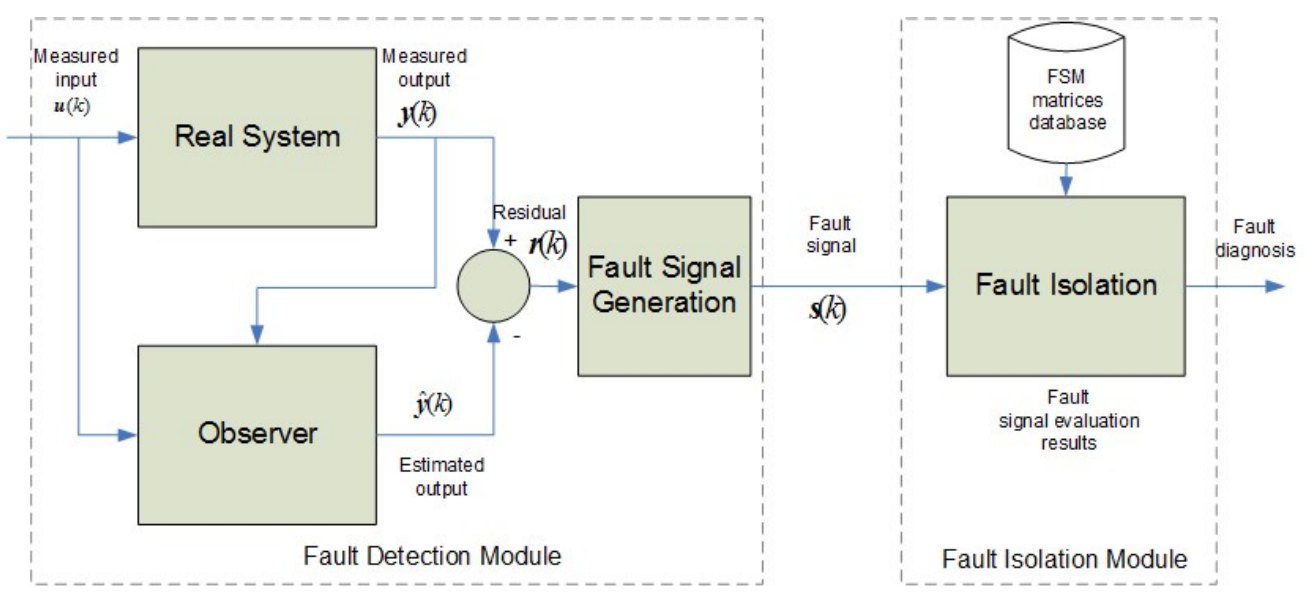

Figure 1: Conceptual diagram of the model-based fault diagnosis approach

$40 \%$

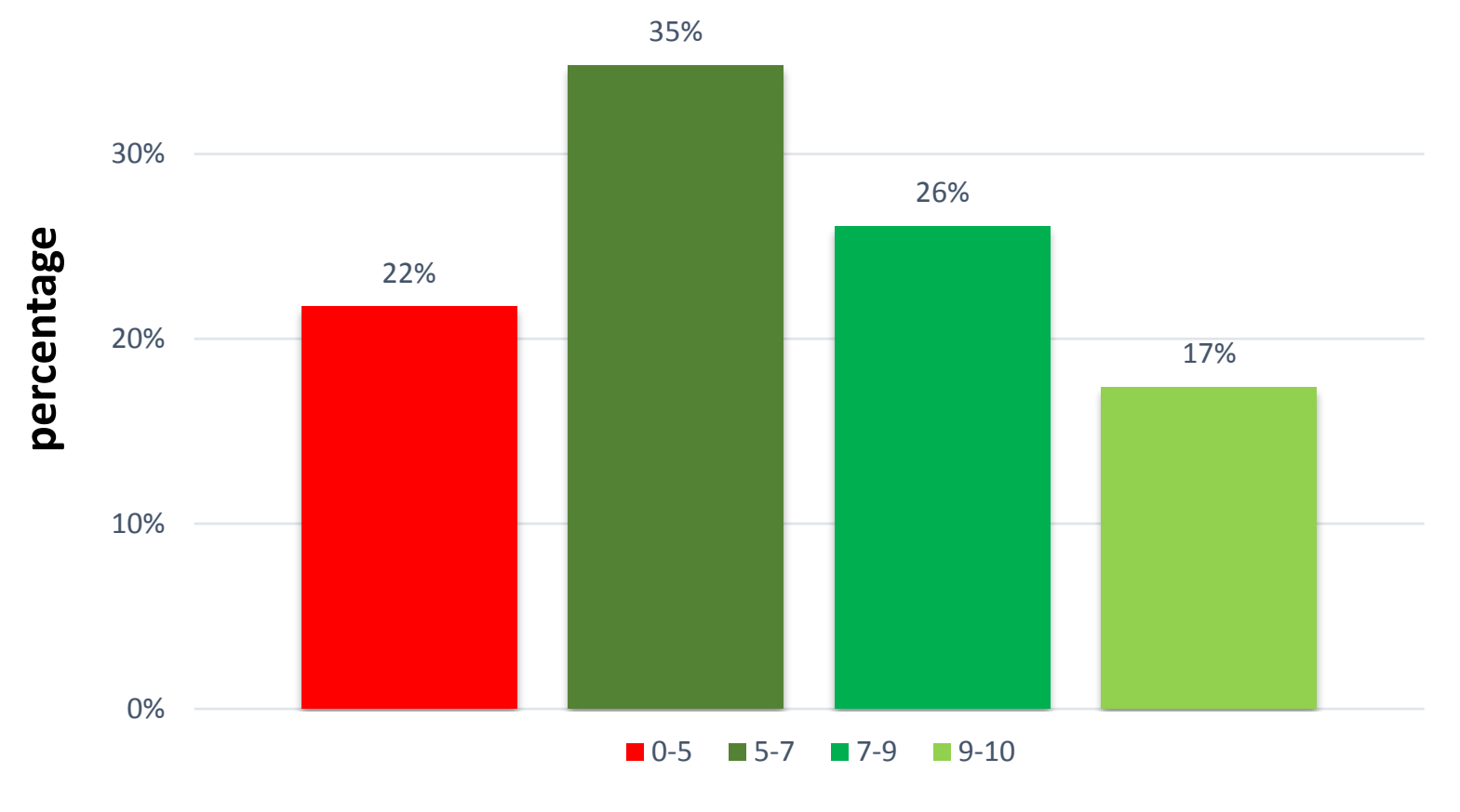

Figure 2: Academic results before the application of PBL methodology in the period 2006-2011: Not satisfactory [0,5), Satisfactory [5,7), Very Good [7-9), Excellent (9-10]. 
students. As a conclusion the proposed methodology, activities, and materials are considered a good approach to introduce fault-diagnosis methods to control engineering students.

\section{Conclusions}

This article presents an example of how a fault-diagnosis course has been included in a engineering curricula in a motivating way. In particular, in the Automatic Control and Robotic Master's degree program at UPC. This article also shows how a PBL methodology has bee used in the lab sessions of the course to introduce students the model-based fault diagnosis field. With this methodology, a real-world problem is used to increase students' interest and at the same time to help them to acquire and apply new knowledge in a problem-solving context. Observer-based and parity-equations-based methods for fault detection and structured residuals for fault isolation are introduced to the students from a practical point of view by means of a set of lab exercises. The course also motivates the interest in fault diagnosis as a first step towards the design of a fault-tolerant control system. Assessment results and student surveys carried out before and after the application of the PBL methodology confirm the increase of interest and fulfillment of the learning objectives.

\section{References}

[1] M. Blanke, M. Kinnaert, J. Lunze, and M. Staroswiecki, Diagnosis and Fault-Tolerant Control. Second Edition. Springer-Verlag Berlin Heidelberg, 2006.

[2] J. Gertler, Fault Detection and Diagnosis in Engineering Systems. New York: Marcel Dekker, 1998.

[3] R. N. Clark, D. Fosth, and V. M. Walton, "Detection instrument malfunctions in control 
systems," IEEE Transactions Aerospace Electronic Systems, vol. 11, pp. 465-473, 1975.

[4] J. Chen and R. Patton, Robust Model-Based Fault Diagnosis for Dynamic Systems. Kluwer Academic Publishers, 1999.

[5] R. Isermann, "Fault diagnosis of machines via parameter estimation and knowledge processing," Automatica, vol. 29, pp. 815-836, 1993.

[6] —-, Fault-Diagnosis Systems: An Introduction from Fault Detection to Fault Tolerance. Berlin, Germany: Springer, 2006.

[7] L. Travé-Massuyès, "Bridging control and artificial intelligence theories for diagnosis: A survey," Engineering Applications of Artificial Intelligence, vol. 27, pp. 1-16, January 2014.

[8] R.Isermann, "Supervision, fault-detection and fault-diagnosis methods - an introduction," Control Engineering Practice, vol. 5, no. 5, p. 639-652, 1997.

[9] —_, "Model-based fault-detection and diagnosis - status and applications," Annual Reviews of Control, vol. 29, no. 1, pp. 71 - 85, 2005.

[10] P. Frank, "Analytical and qualitative model-based fault diagnosis-a survey and some new results," European Journal of Control, vol. 2, no. 1, pp. 6-28, 1996.

[11] P. Frank and X. Ding, "Survey of robust residual generation and evaluation methods in observer-based fault detection systems," Journal of Process Control, vol. 7, no. 6, pp. 403424, 1997.

[12] R. Patton, "Robust model-based fault diagnosis: The state of the art," in Proceedings of the IFAC SAFEPROCESS 1994, Espoo, Finland, 1994, pp. 1-27.

[13] V. Venkatasubramanian, R. Rengaswamy, K. Yin, and S. N. Kavuri, "A review of process fault detection and diagnosis: Part I: Quantitative model-based methods," Computer and Chemical Engineering, vol. 27, no. 3, p. 293-311, 2003. 
[14] — , "A review of process fault detection and diagnosis: Part II: Qualitative models and search strategies," Computer and Chemical Engineering, vol. 27, no. 3, pp. 313-326, 2003.

[15] — , "A review of process fault detection and diagnosis: Part III : Process history based methods," Computer and Chemical Engineering, vol. 27, no. 3, p. 327-346, 2003.

[16] R. J. Patton, P. M. Frank, and R. N. Clark, Fault Diagnosis in Dynamic Systems, Theory and Applications. Englewood Cliffs, New Jersey: Prentice-Hall, 1989.

[17] J. Chen, R. Patton, and Z. Chen, "An LMI approach to fault-tolerant control of uncertain systems," in Proceedings of the International Symposium on Intelligent Control (ISIC). Held jointly with IEEE International Symposium on Computational Intelligence in Robotics and Automation (CIRA), Intelligent Systems and Semiotics (ISAS), Sep 1998, pp. 175-180.

[18] R. Isermann, Fault-Diagnosis Applications. Springer-Verlag, Berlin, Heidelberg, 2011.

[19] S. Ding, Model-based Fault Diagnosis Techniques: Design Schemes, Algorithms, and Tools. Springer-Verlag, Berlin, Heidelberg, 2008.

[20] M. Blanke and T. Lorentz, "Satool: A software tool for structural analysis of complex automation systems," in Proceedings of the 6th IFAC Symposium on Fault Detection, Supervision and Safety of Technical Processes, Beijing, China, 2006, p. 673-678.

[21] A. Varga, "A fault detection toolbox for Matlab," in Proceedings of the 2006 Computer Aided Control System Design, 2006 IEEE International Conference on Control Applications, 2006 IEEE International Symposium on Intelligent Control, Oct 2006, pp. 3013-3018.

[22] — - "A descriptor systems toolbox for Matlab," in IEEE International Symposium on Computer-Aided Control System Design, 2000. CACSD 2000, 2000, pp. 150-155.

[23] S. X. Ding, E. Atlas, S. Schneider, Y. Ma, T. Jeinsch, and E. L. Ding, "An introduction to a Matlab-based FDI-toolbox," in Proceedings of the 6th IFAC Symposium on Fault Detection, 
Supervision and Safety of Technical Processes, Beijing, China, 2006, pp. 651-656.

[24] A. Kumar, S. Fernando, and R. Panicker, "Project-based learning in embedded systems education using an fpga platform," IEEE Transactions on Education, vol. 56, no. 4, pp. 407-415, 2013.

[25] D. G. Lamar, P. F. Miaja, M. Arias, A. Rodríguez, M. Rodríguez, A. Vázquez, M. M. Hernando, and J. Sebastián, "Experiences in the application of project-based learning in a switching-mode power supplies course," IEEE Transactions on Education, vol. 55, no. 1, pp. 69-77, February 2012.

[26] J. Kim, "An ill-structured pbl-based microprocessor course without formal laboratory," IEEE Transactions on Education, vol. 55, no. 1, pp. 145-153, February 2012.

[27] S. Dormido and F. Esquembre, "The quadruple-tank process: An interactive tool for control education," in European Control Conference, 2003.

[28] C. Join, H. Sira-Ramírez, and M. Fliess, "Control of an uncertain three-tank system via on-line parameter identification and fault detection," in Proceedings of the 16th IFAC World Congress, 2005.

[29] K. H. Johansson, "The quadruple-tank process: A multivariable laboratory process with an adjustable zero," IEEE Transacions on Control Systems Technology, vol. 8, no. 3, pp. 456-465, May 2000.

[30] R. Dormido, H. Vargas, N. Duro, J. Sanchez, S. Dormido-Canto, G. Farias, F. Esquembre, and S. Dormido, "Development of a web-based control laboratory for automation technicians: The three-tank system," IEEE Transactions on Education, vol. 51, no. 1, pp. 35-44, Feb 2008.

[31] M. Pasamontes, J. Alvarez, J. Guzman, and M. Berenguel, "Learning switching control: 
A tank level-control exercise," IEEE Transactions on Education, vol. 55, no. 2, pp. 226 232, May 2012.

[32] V. Puig, J. Quevedo, T. Escobet, F. Nejjari, and S. de las Heras, "Passive robust fault detection of dynamic processes using interval models," IEEE Transactions on Control Systems Technology, vol. 16, no. 5, pp. 1083-1089, 2008. 


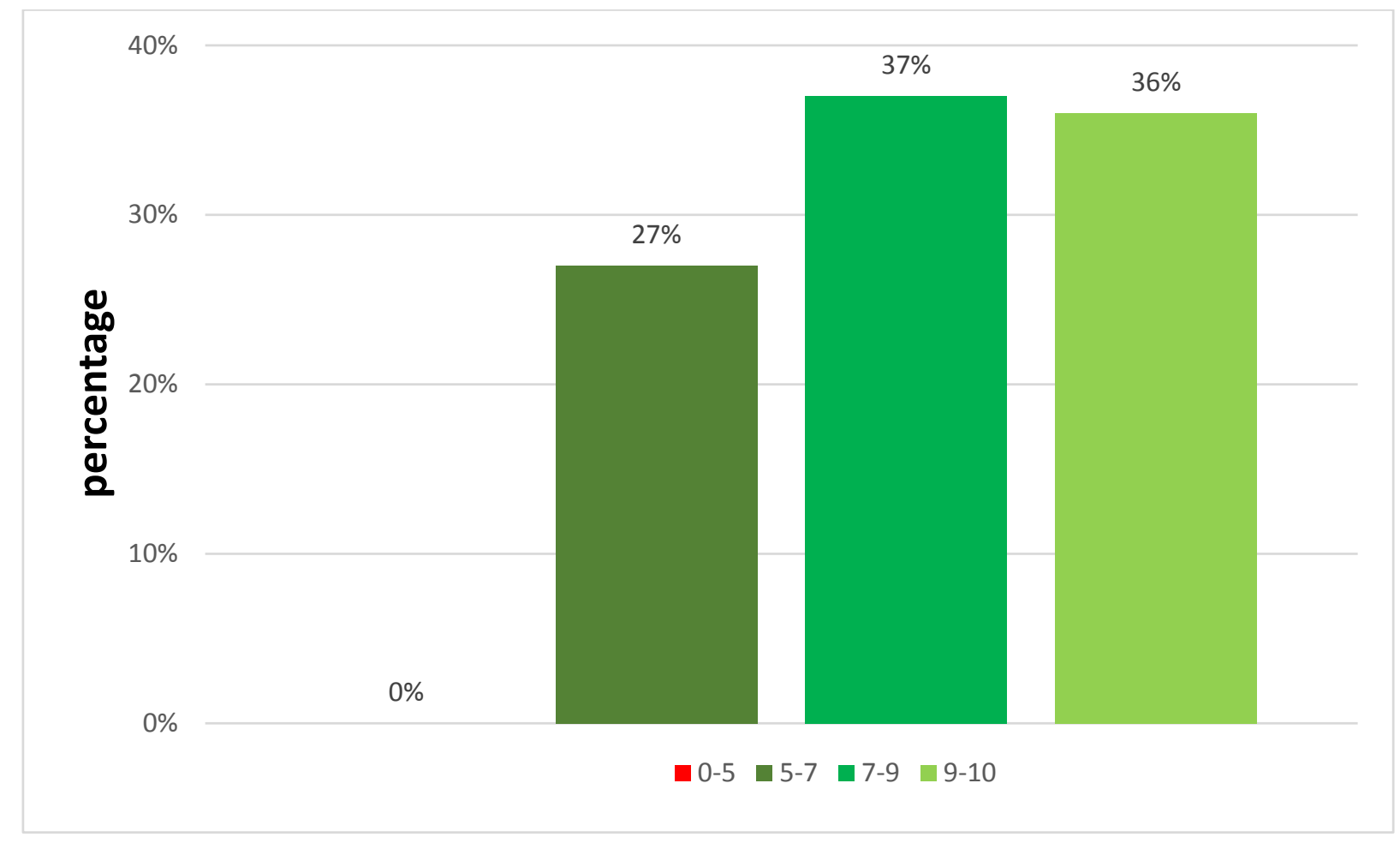

Figure 3: Students' academic results before the application of PBL methodology in the period from academic year 2006/2007 to academic year 2010/2011: Not satisfactory [0,5), Satisfactory [5,7), Very Good [7-9), Excellent (9-10]. 


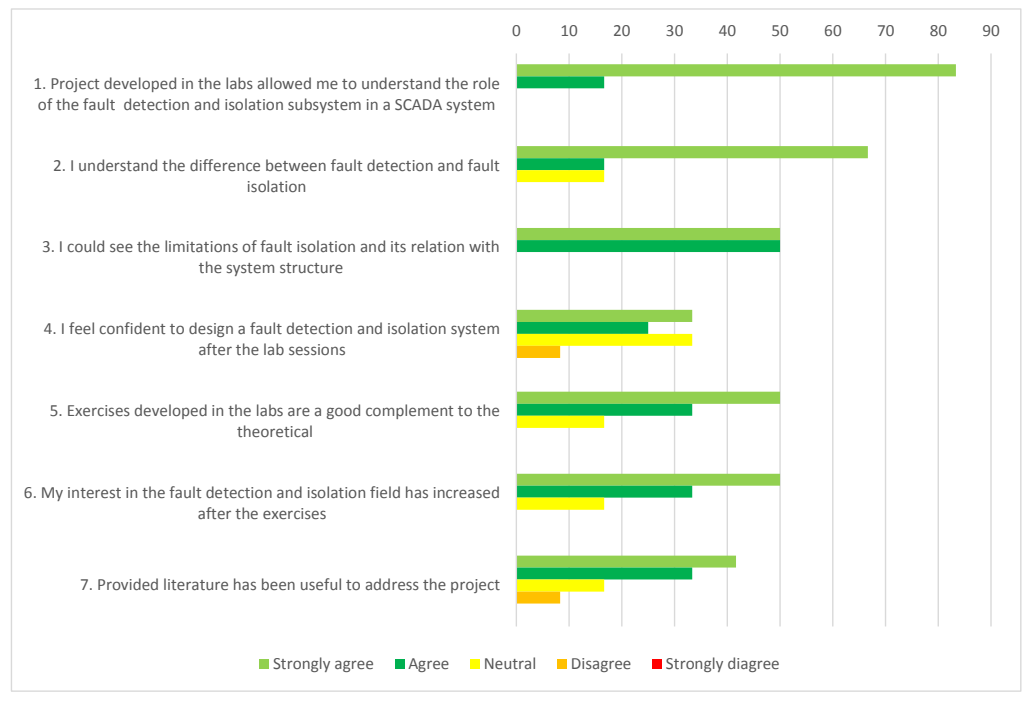

Figure 4: Students' opinion on the fault-diagnosis course exercises in the two academic years (2012-13 and 2011-12 period). 


\section{Sidebar 1: Faults}

Faults should be understood as unpermitted deviations of at least one characteristic property or parameter of the system from the nonimal condition. Faults should be distinguished from failures that correspond to the permanent interruption of the system ability to perform a required function under given specific operating conditions.

Faults can be classified according to several criteria as:

- Temporal evolution: abrupt, incipient or intermittent.

- Type of effect: additive (for example, an offset appears in the sensor output) or multiplicative (for example, a change in a parameter value).

- Location of the faults: sensors, actuators or plant. 
Table I: List of components and associated models of the three tank system

\begin{tabular}{|c|c|}
\hline Component & Model \\
\hline Tank 1 & $c_{1}: \dot{h_{l}}=\frac{1}{a_{l}}\left(q_{p l b}-q_{l b}-q_{l m}\right)$ \\
Tank m & $c_{2}: h_{m}=\frac{1}{a_{m}}\left(q_{l m}-q_{m r}\right)$ \\
Tank r & $c_{3}: \dot{h_{r}}=\frac{1}{a_{r}}\left(q_{p r b}-q_{r b}+q_{m r}\right)$ \\
Pipe lb & $c_{4}: q_{l b}=k_{l b} s\left(h_{l}-h_{b}\right)$ \\
Pipe lm & $c_{5}: q_{l m}=k_{l m} s\left(h_{l}-h_{m}\right)$ \\
Pipe mr & $c_{6}: q_{m r}=k_{m r} s\left(h_{m}-h_{r}\right)$ \\
Pipe rb & $c_{7}: q_{r b}=k_{r b} s\left(h_{r}-h_{b}\right)$ \\
Pump lb & $c_{8}: q_{p l b}=k_{l} u_{l}$ \\
Pump rb & $c_{9}: q_{p r b}=k_{r} u_{r}$ \\
Level sensor 1 & $c_{10}: y_{l}=h_{l}$ \\
Level sensor m & $c_{11}: y_{m}=h_{m}$ \\
Level sensor r & $c_{12}: y_{r}=h_{r}$ \\
\hline
\end{tabular}




\section{Sidebar 2: Fault diagnosis}

Fault diagnosis consists of the determination of the kind, size, location and detection time of a fault by evaluating changes from nominal conditions in system observable quantities. Thus, fault diagnosis involves the following steps:

- Fault detection determines whether a fault is present in the system and provides the time at what its presence has been noticed.

- Fault isolation determines the kind of fault and where it is located.

- Fault estimation determines the size and time-variant behaviour of the fault.

In the case of the three-tank real setup presented in Figure 6, fault detection will allow knowing that some system component (for example, sensor or pump) is not behaving as expected. The fault isolation will allow determining which is the faulty component (for example, which sensor or pump is not working well) and the type of fault (for example, additive or parametric). Finally, the fault estimation allows determining the size of the fault (for example, which is the change in the cross section of the valve or pipe). 


\section{Sidebar 3: Structural analysis and sensor placement}

Structural analysis allows determining the structural properties of system models, that is, properties which are independent of the actual values of the parameters [1]. From the point of view of structural analysis, the model of the system is considered as a set of constraints (model equations) that apply to a set of variables among which a subset have known values (provided by the sensors). In the particular case of the three-tank real setup presented in Figure 6. the constraints are the equations describing the component models (see Table \). The known variables are the input voltage to the pumps and the measured variables are the sensor levels. The system structure is typically described through a graph, where the variables and parameters are nodes and the links are the arcs (see Figure 6).

Structural analysis provides tools that cover several steps of the design of the fault-diagnosis system: determination of extra sensors to improve diagnosis results, determination of residual expressions, evaluation of fault detectability and isolability, among other.

The sensor placement problem aims at deciding which sensors should be installed to satisfy a given set of fault detectability and isolability properties. Structural analysis also provides a framework for solving the sensor placement problem, for example including a flow sensor appropriately would improve the diagnosis capabilities in the three tank system. 


\section{Sidebar 4: Model-based fault detection and isolation}

The principle of model-based fault detection is to test whether the measured inputs and outputs are consistent with the model of the faultless system. If the measurements are inconsistent with the model of the faultless system that proves the existence of a fault. The residual usually describes the consistency check between the predicted, $\hat{y}(k)$ and the real behavior, $y(k)$, as

$$
r(k)=y(k)-\hat{y}(k) .
$$

The fault-detection task consists in deciding if a residual given by Eq. (S1] is violated at a given instant or not generating a fault signal $s_{i}$ according to:

$$
s_{i}= \begin{cases}0, & \text { if }\left|r_{i}(k)\right|<\tau_{i} \text { (no fault), } \\ 1, & \text { if }\left|r_{i}(k)\right| \geq \tau_{i} \text { (fault), }\end{cases}
$$

where $\tau_{i}$ is the threshold associated with the $i$-th residual.

Fault isolation is usually based on designing a vector of structured residuals [2]. Each residual is designed to be sensitive to a subset of faults, while remaining insensitive to the remaining faults. An alternative way of achieving the isolability of faults is to design a vector of directional residuals [2], that lies in a fixed and fault-specified direction in the residual space, in response to a particular fault. The fault-isolation problem consists of determining which of the known fault directions, called fault signatures, the generated residual vector lies the closest to. This is done as follows. The actual fault signature of the system $\mathbf{s}(k)=\left[s_{1}(k), s_{2}(k), \ldots s_{n}(k)\right]$, obtained as a result of the fault detection phase (see $(\underline{\mathbf{S} 2})$ ), is provided to the fault-isolation module, which 
tries to isolate the fault and provide a diagnosis. The actual fault signature is compared against the theoretical FSM. The FSM binary codifies the influence of a fault in a set of considered faults $f_{1}, f_{2}, \ldots f_{n_{f}}$ on every residual in the a set of considered residuals $r_{1}, r_{2}, \ldots r_{n_{r}}$. This matrix has as many rows as residuals and as many columns as considered faults. An element $F S M_{i j}$ of this matrix being equal to 1 means that the $j^{\text {th }}$ fault affects the $i^{\text {th }}$ residual. Otherwise, the element of the FSM is equal to 0. Assuming classical FDI fault hypotheses, that are single faults and no-compensation (exoneration), fault isolation will consist of looking for a column of the FSM that matches the actual fault signature $\mathbf{s}(k)$. Therefore, this classic approach in the FDI-community is also known as column reasoning [2]. 


\section{Sidebar 5: Fault-tolerant control}

Fault-tolerant control (FTC) allows having a control loop that fulfills its objectives (maybe with a possible degradation) even when faults in components of the system (instrumentation, actuators and/or plant) appear. From the point of view of the control strategies, the literature considers two main groups of techniques. First, passive FTC techniques are based on synthesizing a controller so that the closed-loop system is stable for a set of faults defined a priori. On the other hand, active FTC techniques consist of adapting the control loop using the information given by the fault diagnosis module. This information is used to change the controller parameters or structure to avoid the consequences of the fault. This procedure is usually called fault accommodation. Fault accommodation can be used if the fault-diagnosis module provides the fault estimation, since a model of the system including the fault effect can be used and the controller can be redesigned accordingly. Otherwise, if fault estimation is not available, the faulty components should be disconnected and the controller should be redesigned to control the system with the remaining healthy components.

One of the particularly interesting FTC strategies is based on the idea of virtual sensors/actuators that are used in the faulty control loop to hide the fault such that the nominal controller could be still used without need of returning it. The plant with the faulty actuator/sensor is modified by adding the virtual sensor/actuator block that masks the fault and allows the controller to see the same plant as before the fault. 


\section{Sidebar 6: Project-based learning}

The core idea of project-based learning is that real-world problems capture students' interest and motivate serious thinking as the students acquire and apply new knowledge in a problem-solving context. The teacher plays the role of facilitator, working with students to frame worthwhile questions, structuring meaningful tasks, coaching both knowledge development and social skills, and carefully assessing what students have learned from the experience.

Applying a PBL methodology in a course, as the one described in this article, requires that the teacher plays a very active role to guide the students' steps otherwise they expend many time discussing inappropriate approaches. Although these discussions are also very formative makes that most students will not complete the experimental work (that usually requires restrictive access to the lab) within the expected time window. 


\section{Author Information}

Ramon Costa-Castelló received the master degree in computer science in 1993 from the Universitat Politècnica de Catalunya (UPC), in 2001 he received the $\mathrm{PhD}$ degree in computer science from the UPC. He is an Associate Professor at the Automatic Control department of the UPC. His teaching activity is with the Industrial Engineering and the master in automation and robotics (MAR), both from the School of Industrial Engineering of Barcelona (ETSEIB). His research is mainly focused on analysis and development of energy management (automotive and stationary applications) and the development of digital control techniques for tracking/rejection periodic signals (repetitive control, resonant control), with application to power electronic converters (active filters, rectifiers) and mechatronic plants. In parallel with this activity he works on the development of virtual/remote laboratories and interactive applications applied to teaching automatic control. He is a member of the Comité Español de Automática (CEA) and member of IFAC (TC 9.4 Committee, TC 7.1 Committee).

Vicenç Puig received the the Telecommunications Engineering degree in 1993 and the $\mathrm{PhD}$ degree in Automatic Control, Vision and Robotics in 1999, both from Universitat Politècnica de Catalunya (UPC). He is an Associate Professor at the Automatic Control department of the UPC and researcher at the Institut de Robòtica i Informàtica Industrial, CSIC-UPC. He is the leader of the research group in Advanced Control Systems (SAC) at the UPC. He has developed important scientific contributions in the areas of fault diagnosis and fault tolerant control using interval and LPV models. He has participated in more than 20 European and national research projects in the last decade. He has also led many private contracts with several companies, and has published more than 80 articles in JCR journals and more than 350 in international conference/workshop proceedings. He has supervised over $15 \mathrm{PhD}$ theses and over 40 master 
theses/final projects. He is currently the vice-chair of the IFAC SAFEPROCESS TC Committee 6.4 in the period 2014-2017. He will be chairman of 3rd IEEE Conference on Control and Fault-Tolerant Systems (Systol'2016) organized at UPC in 2016 in Barcelona (Spain). And, he also will be the IPC chair of the IFAC Safeprocess 2018 organized in Poland.

Joaquim Blesa received the Telecommunications Engineering degree in 1997 and $\mathrm{PhD}$ degree in Control, Vision and Robotics in 2011 both from Universitat Politècnica de Catalunya (UPC), Barcelona, Spain. He is currently an Assistant Professor of Automatic Control at UPC and researcher in the Research Center for Supervision, Safety and Automatic Control (CS2AC) at UPC and in the Institut de Robòtica i Informàtica Industrial, CSIC-UPC. His current research interests include robust identification in the automatic control field and the fault diagnosis of dynamic systems. He has published several papers in journals and international conferences and participated in several European projects and networks related with these topics. 


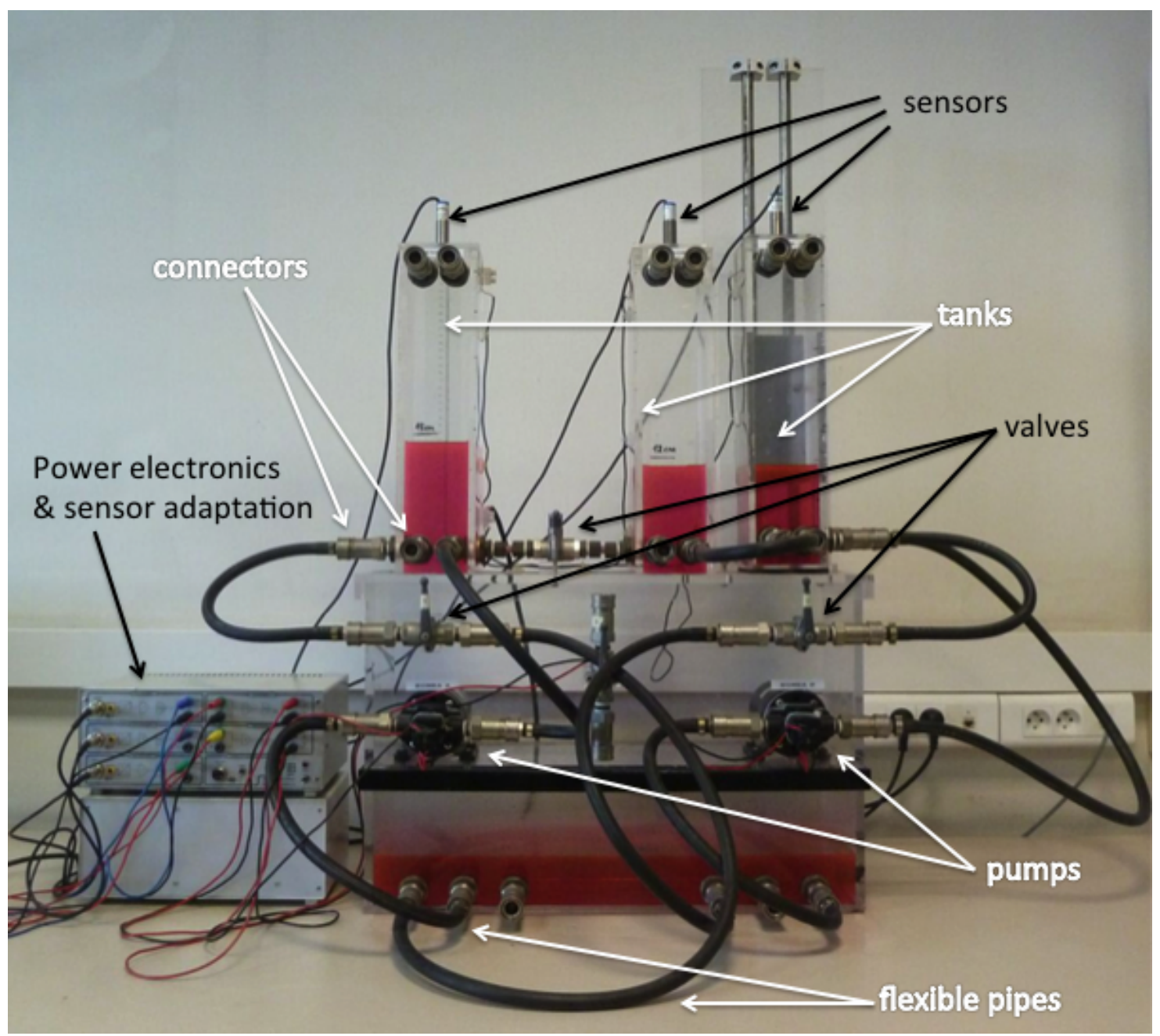

Figure 5: The three-tank real experimental setup presenting the different components. 


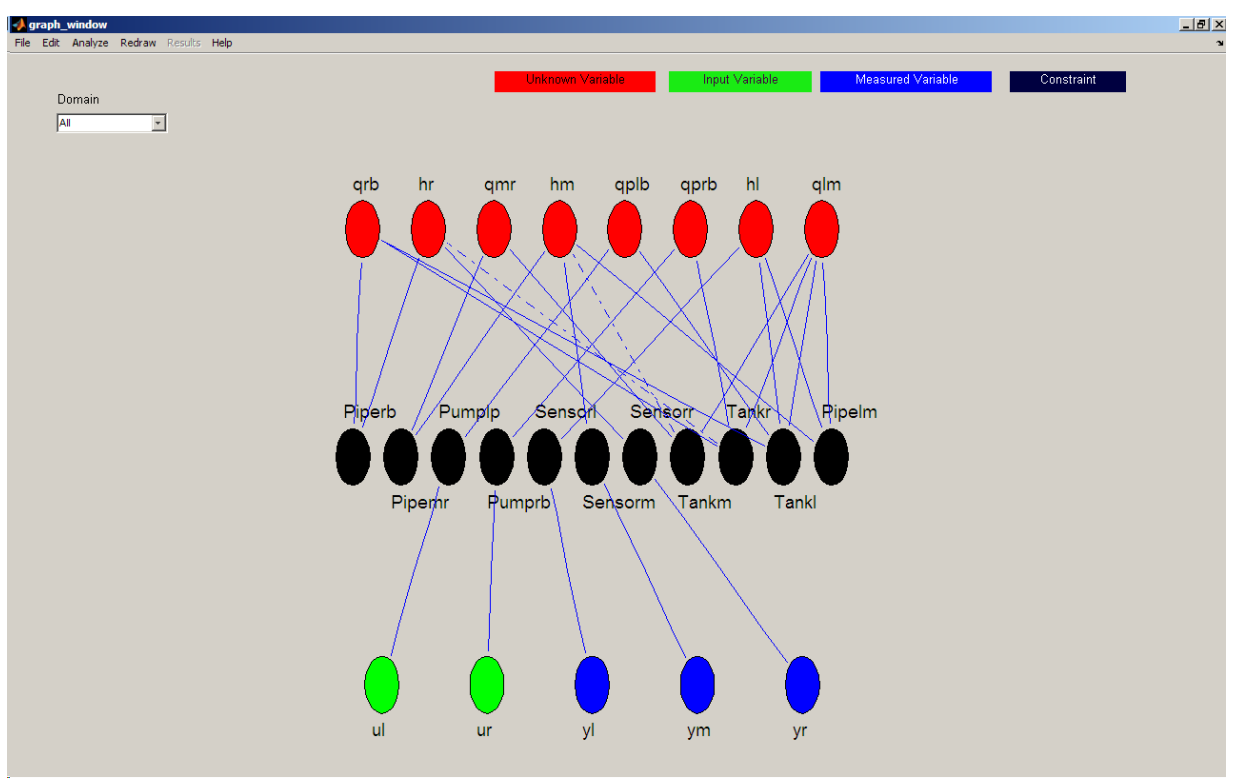

Figure 6: Structural analysis graph obtained using SaTool. The figure is a direct snapshot screen capture of the SaTool analysis window. The constraints are presented in black, the measured variables in blue, the input variables in green and the unknown variables in red. 

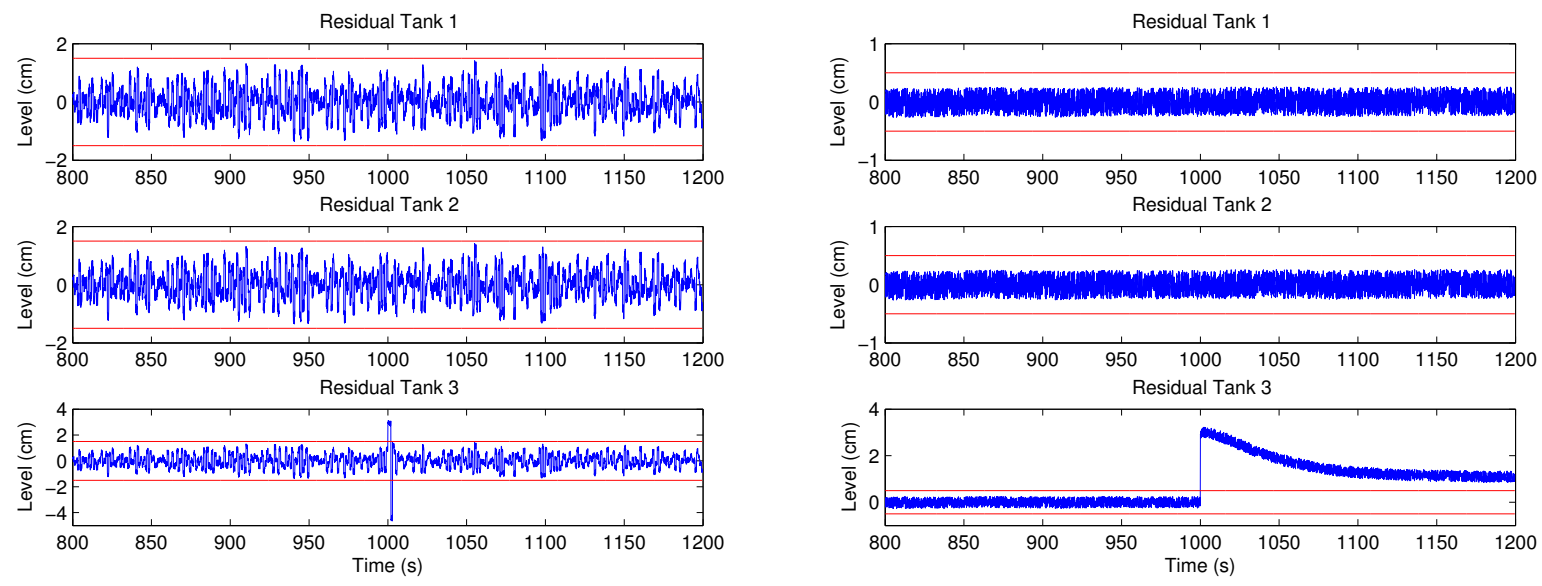

(a) MA parity equations

(b) ARMA parity equations

Figure 7: Effect of the observer gain in fault detection: In the case of the MA parity equations the fault is indicated only at the appearance time (because of the moving average strategy) while in the ARMA parity the fault is more persistently indicated (because of the autoregressive strategy). The most persistent indication will be achieved when the observer gain is zero that corresponds to the case that residuals are implemented in simulator form. Unfortunately, this case is not always applicable as e.g. in the case that not all state are measurable. 
Table II: Fault signature matrix of the three-tank system considering faults in level sensors $\left(f_{y l}\right.$ , $f_{y m}$ and $\left.f_{y r}\right)$ and pumps $\left(f_{u l}\right.$ and $\left.f_{u r}\right) . r_{1}, r_{2}$ and $r_{3}$ are the residuals obtained by means the matching algorithm (structural analysis graph in Figure 6) to the set of constraints presented in Table II. It can be noticed from this table that sensor faults are not isolable.

\begin{tabular}{|c||c|c|c|c|c|}
\hline \multicolumn{1}{|c||}{ Residual } & $f_{y_{l}}$ & $f_{y_{m}}$ & $f_{y_{r}}$ & $f_{u_{l}}$ & $f_{u_{r}}$ \\
\hline \hline$r_{1}$ & 1 & 1 & 1 & 1 & 0 \\
$r_{2}$ & 1 & 1 & 1 & 0 & 0 \\
$r_{3}$ & 1 & 1 & 1 & 0 & 1 \\
\hline
\end{tabular}


Table III: Structured fault signature matrix of the three-tank system using the method proposed in [2], where each individual residual $\left(r_{l}, r_{m}\right.$ and $\left.r_{r}\right)$ is designed to be sensitive to a single sensor fault $\left(f_{y l}, f_{y m}\right.$ and $\left.f_{y r}\right)$ while remaining insensitive to the rest of the sensor faults.

\begin{tabular}{|c||c|c|c|c|c|}
\hline \multicolumn{1}{|c||}{ Residual } & $f_{y_{l}}$ & $f_{y_{m}}$ & $f_{y_{r}}$ & $f_{u_{l}}$ & $f_{u_{r}}$ \\
\hline \hline \multicolumn{1}{|c||}{$r_{l}$} & 1 & 0 & 0 & 1 & 1 \\
$r_{m}$ & 0 & 1 & 0 & 1 & 1 \\
$r_{r}$ & 0 & 0 & 1 & 1 & 1 \\
\hline
\end{tabular}


Authors: Ramon Costa-Castelló, Vicenç Puig and Joaquim Blesa

Afilliation :

Automatic Control Department (ESAII)

Universitat Politècnica de Catalunya (UPC)

Pau Gargallo, 5,

08028 Barcelona. 